\title{
MONOTONICITY OF NONLINEAR BOUNDARY VALUE PROBLEMS RELATED TO DEFORMATION THEORY OF PLASTICITY
}

\author{
ALEMDAR HASANOV
}

Received 1 March 2005; Accepted 20 June 2005

We study nonlinear boundary value problems arising in the deformation theory of plasticity. These problems include 3D mixed problems related to nonlinear Lame system, elastoplastic bending of an incompressible hardening plate, and elastoplastic torsion of a bar. For all these different problems, we present a general variational approach based on monotone potential operator theory and prove solvability and monotonicity of potentials. The obtained results are illustrated on numerical examples.

Copyright (c) 2006 Alemdar Hasanov. This is an open access article distributed under the Creative Commons Attribution License, which permits unrestricted use, distribution, and reproduction in any medium, provided the original work is properly cited.

\section{Introduction}

We present a general variational approach for boundary value problems related to deformation theory of plasticity, based on the theory of monotone potential operators. The fundamentals of deformation theory of plasticity for homogeneous isotropic deformable materials have been given in $[9,10]$, and then developed by many authors $[2,4,15]$. According to the main assumption of the deformation theory, if a loading is small and close to simple loading, then the relationship between strain $e_{\mathrm{i}}$ and stress $\sigma_{\mathrm{i}}$ intensities can be described by the function (deformation curve, Figure 1.1)

$$
\sigma_{\mathrm{i}}=\sigma_{\mathrm{i}}\left(e_{\mathrm{i}}\right) \text {, }
$$

which satisfies the following conditions:

(a1) $\sigma_{\mathrm{i}} \in C^{1}\left(0, e_{\mathrm{i}}^{*}\right) ; d \sigma_{\mathrm{i}} / d e_{\mathrm{i}}>0$ (monotonicity),

(a2) $d \sigma_{\mathrm{i}} / d e_{\mathrm{i}}>\sigma_{\mathrm{i}} / e_{\mathrm{i}} \leq 0$, for all $e_{\mathrm{i}} \in\left(0, e_{\mathrm{i}}^{*}\right)$ (concavity),

(a3) there exist $e_{\mathrm{i}}^{0} \in\left(0, e_{\mathrm{i}}^{*}\right), \sigma_{\mathrm{i}}\left(e_{\mathrm{i}}\right)=3 G e_{\mathrm{i}}$, for all $e_{\mathrm{i}} \in\left(0, e_{\mathrm{i}}^{0}\right)$ (pure elastic deformations). 


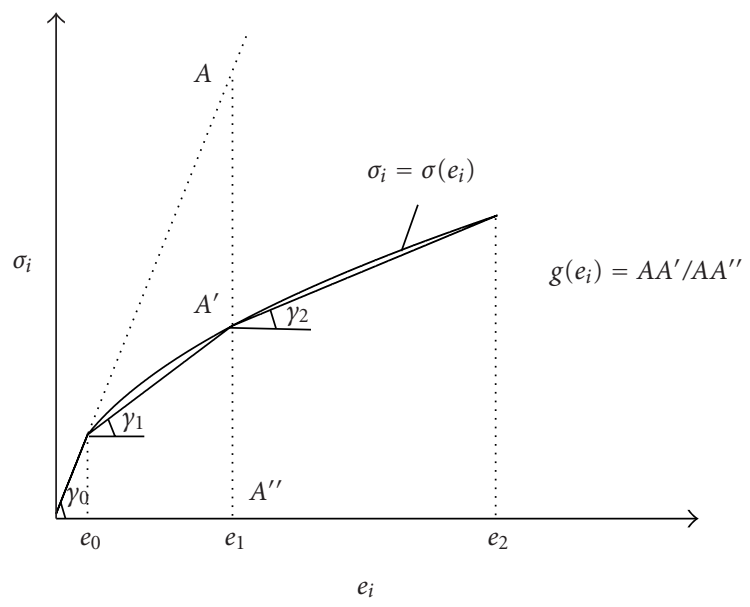

Figure 1.1. The deformation curve.

Here,

$$
\begin{aligned}
& e_{\mathrm{i}}(u)=\left\{\left(\frac{2}{3}\right) e_{i j}(u) e_{i j}(u)\right\}^{1 / 2}, \quad e_{i j}(u)=\varepsilon_{i j}(u)-\theta(u) \delta_{i j}, \\
& \theta(u)=\frac{1}{3}\left[\varepsilon_{11}(u)+\varepsilon_{22}(u)+\varepsilon_{33}(u)\right], \quad \varepsilon_{i j}(u)=\frac{1}{2}\left(\frac{\partial u_{i}}{\partial x_{j}}+\frac{\partial u_{j}}{\partial x_{i}}\right),
\end{aligned}
$$

$\mathcal{E}(u)=\left\{\varepsilon_{i j}(u)\right\}$ and $e(u)=\left\{e_{i j}(u)\right\}$ are tensor and deviator of deformation, respectively, $G=\mu$ modulus of rigidity, $e_{\mathrm{i}}^{0}>0$ elasticity limit of a deformable material, $u(x)=\left(u_{1}\left(x_{1}\right.\right.$, $\left.\left.x_{2}, x_{3}\right), u_{2}\left(x_{1}, x_{2}, x_{3}\right), u_{3}\left(x_{1}, x_{2}, x_{3}\right)\right)$ is a displacement vector and $\delta_{i j}$-Kronecker delta (the summation convention from 1 to 3 will be employed throughout).

We denote by $A$ a nonlinear differential operator related to a plasticity problem, and assume that $A$ is defined on a Hilbert space $H$. Let $a(u ; u, v)$ be the corresponding bounded nonlinear functional defined on $H \times H \times H$, that is,

$$
\begin{gathered}
\langle A u, v\rangle=a(u ; u, v), \quad \forall u, v \in H, \\
|a(u ; v, w)| \leq \gamma_{2}\|v\|_{H}\|w\|_{H}, \quad \forall v, w \in H, \gamma_{2}>0 .
\end{gathered}
$$

Then the weak solution $u \in H$ of the nonlinear operator equation

$$
A u=F, \quad u \in H, F \in H^{*},
$$

will be defined as follows:

$$
a(u ; u, v)=l(v), \quad \forall v \in H
$$

where $l(v)=\langle F, v\rangle$. We say that the operator $A$ is a potential one if there exists a functional $J(u): H \rightarrow \mathbb{R}$ such that

$$
\left\langle J^{\prime}(u), v\right\rangle=a(u ; u, v), \quad \forall u, v \in H .
$$


The functional

$$
\Pi(u)=J(u)-l(u), \quad u \in H,
$$

will be defined as a potential of the nonlinear problem (1.4).

According to the Browder-Minty theorem [5, 16], if a potential operator is radially continuous (hemicontinuous), strongly monotone, and coercive, then the nonlinear problem (1.4) (or (1.5)) has a unique solution $u \in H$. The first main result of this study is that the monotonicity and concavity conditions (a1)-(a2) imply strong monotonicity of the plasticity operators of all the considered three problems related to nonlinear Lame system, elastoplastic bending of an incompressible hardening plate, and elastoplastic torsion of a bar. As a result, it is proved that these physical assumptions are exacly sufficient for existence and uniqueness of solutions of the above problems. Hence, the BrowderMinty theorem can be extended to the nonlinear boundary value problems arising in the deformation theory.

For the considered class of nonlinear operators, the functional $a(u ; \cdot, \cdot)$ is a symmetric bilinear form defined on $H \times H$. Taking into account this circumstance, we linearize the nonlinear problem (1.5), according to [7], as follows:

$$
a\left(u^{(n-1)} ; u^{(n)}, v\right)=l(v), \quad \forall v \in H, n=1,2,3, \ldots,
$$

where $u^{0} \in H$ is an initial iteration. It is proved in [9] that if the convexity argument

$$
0.5 a(u ; v, v)-0.5 a(u ; u, u)-J(v)+J(u) \geq 0, \quad \forall u, v \in H,
$$

is satisfied for the monotone potential operator $A$, then the following convergence estimate holds:

$$
\left\|u-u^{(n)}\right\|_{H} \leq \frac{\sqrt{2} \gamma_{2}}{\gamma_{1}^{3 / 2}}\left[\Pi\left(u^{(n-1)}\right)-\Pi\left(u^{(n)}\right)\right]^{1 / 2}, \quad \gamma_{1}, \gamma_{2}>0,
$$

and moreover the sequence of potentials $\left\{\Pi\left(u^{(n)}\right)\right\}$ is a monotone decreasing one. Here $\gamma_{1}>0$ is a monotonicity constant, that is,

$$
\langle A(u)-A(v), u-v\rangle \geq \gamma_{1}\|u-v\|_{H}, \quad \forall u, v \in H,
$$

and $\gamma_{2}>0$ is the above boundedness constant. As a second important result, we prove that the concavity condition (a2) is a sufficient condition for the fulfillment of the convexity argument (1.9). As a result, we show monotonicity of the iteration scheme and obtain the same sufficient condition for convergence of the iteration process, for all the above nonlinear problems.

The organization of the remaining part of this paper is as follows. In Section 2, we review the deformation theory of plasticity and describe some important properties of plasticity function $g=g\left(e_{\mathrm{i}}^{2}\right)$. In Section 3, we prove that the conditions (a1)-(a3) are sufficient for potentialness and monotonocity of nonlinear operators of the deformation theory of plasticity. As a result, by the Browder-Minty theorem, we obtain existence theorems for all the considered nonlinear problems. Section 4 is devoted to linearization of 
nonlinear problems. We prove that the concavity and monotonocity conditions (a1)-(a2) are sufficient for fulfillment of the convexity argument (1.9). Based on this result, we construct a monotone iteration scheme for all nonlinear problems and prove its convergence.

\section{Physical model}

By the way of introduction, we review Il'yušin-Kachanov model of deformation theory of plasticity which will be used here. First, we consider elastoplastic deformation of 3D homogeneous isotropic deformable material. In this model, one seeks the unknown displacement vector $u(x)=\left(u_{1}(x), u_{2}(x), u_{3}(x)\right)$, satisfying the following nonlinear boundary value problem in $\mathbb{R}^{3}$ :

$$
\begin{aligned}
-\sigma_{i j, i}(u) & =F(x), \quad x \in \Omega \subset \mathbb{R}^{3}, \\
u(x) & =0, \quad x \in \Gamma_{0} \subset \partial \Omega, \\
\sigma_{i j}(u) n_{j} & =f_{i}(x), \quad x \in \Gamma_{1} \subset \partial \Omega,
\end{aligned}
$$

where $F(x)=\left(F_{1}(x), F_{2}(x), F_{3}(x)\right)$ and $f(x)=\left(f_{1}(x), f_{2}(x), f_{3}(x)\right)$ are source and surface vectors, respectively, $\Gamma_{0} \cup \Gamma_{1}=\varnothing, \overline{\Gamma_{0} \cap \Gamma_{1}}=\partial \Omega, \Gamma_{0} \neq \varnothing$, and $n=\left(n_{1}, n_{2}, n_{3}\right)$ is the unit outward normal vector to the smooth surface $\Gamma_{1}$ of the body $\Omega \subset \mathbb{R}^{3}$. According to this theory, the elastoplastic properties of a material under a simple loading can be described by the deformation curve (1.1) in the form

$$
\sigma_{\mathrm{i}}=3 G\left[1-g\left(e_{\mathrm{i}}^{2}\right)\right] e_{\mathrm{i}},
$$

which satisfies the conditions (a1)-(a3). The function $g=g\left(e_{\mathrm{i}}^{2}\right)$, characterizing degree of plastic deformation of a material, is called the plasticity function (Figure 1.1). The case $g\left(e_{\mathrm{i}}^{2}\right)=0$ corresponds to pure elastic deformations, and in this case, we have only linear part of the deformation curve (1.1). The assumptions (a1)-(a3) imply some important properties of the piecewise differentiable function $g=g(\xi) \in C^{1}{ }_{p}\left(0, \xi^{*}\right), \xi=e_{\mathrm{i}}^{2}$ (see, $[10,11]$ ):

(i) $0<g(\xi)<1$, for all $\xi \in\left(\xi^{0}, \xi^{*}\right)$; $g(\xi)=0$, for all $\xi \in\left(0, \xi^{0}\right)$;

(ii) $1>g(\xi)+2 g^{\prime}(\xi) \xi \geq 0$, for all $\xi \in\left(\xi^{0}, \xi^{*}\right)$;

(iii) $g^{\prime}(\xi)>0$, for all $\xi \in\left(\xi^{0}, \xi^{*}\right)$.

Note that $\xi^{0}>0$ corresponds to the elasticity limit $\xi^{0}=\left(e_{\mathrm{i}}^{0}\right)^{2}$.

The relationship between the stress tensor $\sigma=\left\{\sigma_{i j}\right\}$ and deviator of deformation $e=$ $\left\{e_{i j}\right\}$ is given as follows:

$$
\sigma_{i j}(u)=K \theta(u) \delta_{i j}+2 G\left[1-g\left(e_{\mathrm{i}}^{2}(u)\right)\right] e_{i j}(u), \quad i, j=1,2,3,
$$

or, by using the relationship $e_{i j}(u)=\varepsilon_{i j}(u)-\theta(u) \delta_{i j}$,

$$
\sigma_{i j}(u)=\left[K-\frac{2}{3} G\left[1-g\left(e_{\mathrm{i}}^{2}(u)\right)\right]\right] \theta(u) \delta_{i j}+2 G\left[1-g\left(e_{\mathrm{i}}^{2}(u)\right)\right] \varepsilon_{i j}(u), \quad i, j=1,2,3 .
$$

Here $K=\lambda+(2 / 3) \mu$ is the modulus of volumetric expansion, $G=\mu$ is the modulus of rigidity, and $\lambda>0$ and $\mu>0$ are the Lame constants. By using relationship (2.4), we can 
rewrite the nonlinear Lame system (2.1) in the form of the plasticity operator

$$
A u \equiv-(\lambda+2 \mu) \operatorname{grad} \operatorname{div} u-2 \mu \Delta u+2 \mu \operatorname{div}\left[g\left(e_{\mathrm{i}}^{2}\right) e(u)\right]=F(x), \quad x \in \Omega,
$$

in terms of independent variables displacements.

Consider now the elastoplastic deformation of a plate with the thickness $l>0$. According to the deformation theory of plasticity, the stress-strain relationship between deviators of deformation $\varepsilon^{D}=\left\{\varepsilon_{i, j}^{D}\right\}$ and stress $\sigma^{D}=\left\{\sigma_{i, j}^{D}\right\}, i, j=1,2,3$, is given by the Hencky correlation (see, $[10,17])$ :

$$
\sigma_{i, j}^{D}=2 \widetilde{g}\left(\Gamma^{2}\right) \varepsilon_{i, j}^{D}, \quad i, j=1,2,3
$$

As a result, the relationship between the intensities of shift strain $\Gamma=\left(2 \varepsilon_{i j}^{D} \varepsilon_{i, j}^{D}\right)^{1 / 2}$ and tangential stress $\widetilde{T}=\left(2 \sigma_{i, j}^{D} \sigma_{i, j}^{D}\right)^{1 / 2}$ is obtained by the following formula:

$$
\widetilde{T}=\tilde{g}\left(\Gamma^{2}\right) \Gamma
$$

Let us assume that the plane $O x_{1} x_{2}$ is a middle plane for the considered plate in the coordinate system $O x_{1} x_{2} x_{3}$. In this case, the intensity of shift strain is defined as follows:

$$
\Gamma^{2}=4 x_{3}^{2}\left[\left(\frac{\partial^{2} u}{\partial x_{1}^{2}}\right)^{2}+\left(\frac{\partial^{2} u}{\partial x_{2}^{2}}\right)^{2}+\left(\frac{\partial^{2} u}{\partial x_{1} \partial x_{2}}\right)^{2}+\frac{\partial^{2} u}{\partial x_{1}^{2}} \frac{\partial^{2} u}{\partial x_{2}^{2}}\right]
$$

where $u=u\left(x_{1}, x_{2}\right)$ is the deflection of the middle surface of the plate and $x_{3}$ is the coordinate, which is perpendicular to the plate. We now introduce the dependent variable $\xi=\xi(u)$,

$$
\xi^{2}(u)=\left(\frac{\partial^{2} u}{\partial x_{1}^{2}}\right)^{2}+\left(\frac{\partial^{2} u}{\partial x_{2}^{2}}\right)^{2}+\left(\frac{\partial^{2} u}{\partial x_{1} \partial x_{2}}\right)^{2}+\frac{\partial^{2} u}{\partial x_{1}^{2}} \frac{\partial^{2} u}{\partial x_{2}^{2}},
$$

and following [12], employ the average function

$$
g\left(\xi^{2}\right)=\frac{4}{D} \int_{-l / 2}^{l / 2} \tilde{g}\left(4 x_{3}^{2} \xi^{2}\right) d x_{3}, \quad D=\frac{l^{3}}{3},
$$

instead of the plasticity function $\tilde{g}=\tilde{g}\left(\xi^{2}\right)$. The function $g=g\left(\xi^{2}\right)$ describes the elastoplastic behavior of a deformable plate and usually is called the modulus of plasticity. In terms of these transformations, the general relationship (2.7) can be rewriten as follows:

$$
T=g\left(\xi^{2}\right) \xi, \quad \xi \in\left[0, \xi_{M}\right] .
$$

The dependence (2.11) is determined from experiment, and describes the stress-strain state of increasingly hardening materials. For real materials, the function $T=T(\xi)$, as the function $\sigma_{\mathrm{i}}=\sigma_{\mathrm{i}}\left(e_{\mathrm{i}}\right)$, given by (1.1), is a concave and monotone increasing one: $d T / d \xi \geq$ $\alpha_{1}>0$ (Figure 2.1). In the case of pure elastic deformations, $T=G \xi$, where $G>0$ is the modulus of rigidity. These properties imply the following bounds, similar to (i)-(iii), for 


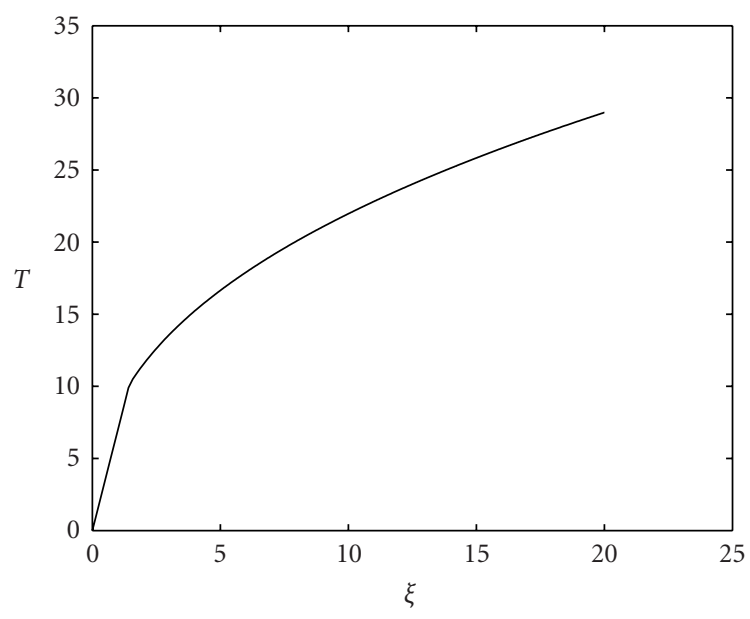

Figure 2.1. The function $T=g\left(\xi^{2}\right) \xi$.

the function $g=g\left(\xi^{2}\right)$ :

(i1) $c_{0} \leq g\left(\xi^{2}\right) \leq c_{1}$;

(i2) $g\left(\xi^{2}\right)+2 g^{\prime}\left(\xi^{2}\right) \xi^{2} \geq c_{2}$;

(i3) $g^{\prime}\left(\xi^{2}\right) \leq 0$, for all $\xi \in\left[0, \xi_{M}\right]$;

(i4) there exist $\xi_{0} \in\left(0, \xi_{M}\right), g\left(\xi^{2}\right)=G$, for all $\xi \in\left[0, \xi_{0}\right]$,

where $c_{i}$ are positive constants. Indeed, condition (i2) follows from the monotonicity condition $d T / d \xi \geq \alpha_{1}>0$; condition (i3) follows from the concavity of function (2.7), since the slope of the tangent is smaller than the slope of the secant, that is,

$$
g^{\prime}\left(\xi^{2}\right)=\frac{1}{2 \xi^{2}}\left(\frac{d T}{d \xi}-\frac{T}{\xi}\right) \leq 0
$$

Finally, condition (i4) means that elastic deformations precede plastic one.

Thus within the range of the above theory, the equation of elastoplastic bending problem for an incompressible strain hardening plate with rigid clamped boundary takes the form [12]

$$
\begin{gathered}
A u \equiv \frac{\partial^{2}}{\partial x_{1}^{2}}\left[g\left(\xi^{2}(u)\right)\left(\frac{\partial^{2} u}{\partial x_{1}^{2}}+\frac{1}{2} \frac{\partial^{2} u}{\partial x_{2}^{2}}\right)\right]+\frac{\partial^{2}}{\partial x_{1} \partial x_{2}}\left[g\left(\xi^{2}(u)\right)\left(\frac{\partial^{2} u}{\partial x_{1} \partial x_{2}}\right)\right] \\
+\frac{\partial^{2}}{\partial x_{2}^{2}}\left[g\left(\xi^{2}(u)\right)\left(\frac{\partial^{2} u}{\partial x_{2}^{2}}+\frac{1}{2} \frac{\partial^{2} u}{\partial x_{1}^{2}}\right)\right]=F(x), \quad x=\left(x_{1}, x_{2}\right) \in \Omega \subset \mathbb{R}^{2}, \\
u(x)=0, \quad \frac{\partial u(x)}{\partial n}=0, \quad x \in \partial \Omega
\end{gathered}
$$

where $\Omega$ is a bounded domain occupied by the middle surface of a plate with a piecewise smooth boundary $\partial \Omega$, and $F(x)=q(x) / D$ is a value proportional to the external normal load $q=q(x)$. 
Finally, consider the problem of elastoplastic torsion of a cylindrical bar $[10,13]$. The governing equation of torsional elastoplastic deformation of the cylindrical bar with rigid clamped torsional session has the form

$$
\begin{gathered}
A u \equiv-\frac{\partial}{\partial x_{1}}\left[g\left(\xi^{2}\right) \frac{\partial u}{\partial x_{1}}\right]-\frac{\partial}{\partial x_{2}}\left[g\left(\xi^{2}\right) \frac{\partial u}{\partial x_{2}}\right]=2 \Theta(x), \quad x=\left(x_{1}, x_{2}\right) \in \Omega \subset \mathbb{R}^{2}, \\
u(x)=0, \quad x \in \partial \Omega,
\end{gathered}
$$

where $\Omega$ is the cross-section of the bar, $\Theta$ is the angle of twist per unit length, $u=u\left(x_{1}, x_{2}\right)$ is Prandtl's stress function, and

$$
\xi(u)=\left[\left(\frac{\partial u}{\partial x_{1}}\right)^{2}+\left(\frac{\partial u}{\partial x_{2}}\right)^{2}\right]^{1 / 2} \equiv|\nabla u|^{2}
$$

is the stress intensity. The stress-strain relationship between deviators is again described by the Hencky correlation (2.11) and the plasticity function satisfies the conditions (i1)(i4).

Thus in all the above nonlinear boundary value problems, the coefficient $g=g\left(\xi^{2}\right)$ satisfies either conditions (i)-(iii), or conditions (i1)-(i4), which follow from the monotonicity and concavity conditions (a1)-(a3).

\section{Potentialness and monotonicity of plasticity operators: existence theorems}

First, we consider the nonlinear problem (2.1)-(2.5). Let us define a weak solution $u \in$ $\stackrel{0}{H}^{1}(\Omega)$ of this problem, multiplying both sides of $(2.5)$ to the function $v \in \stackrel{0}{H}^{1}(\Omega)$, integrating on $\Omega$, and using the boundary conditions (2.1).

Find $u \in \stackrel{0}{H}^{1}(\Omega)$ such that

$$
\begin{gathered}
\int_{\Omega}\left\{\left[\lambda+\frac{2}{3} \mu g\left(e_{\mathrm{i}}^{2}(u)\right)\right] \theta(u) \theta(v)+2 \mu\left[1-g\left(e_{\mathrm{i}}^{2}(u)\right)\right] \varepsilon_{i j}(u) \varepsilon_{i j}(v)\right\} d x \\
=\int_{\Omega} F_{i}(x) v_{i}(x) d x+\int_{\Gamma_{1}} f_{i}(x) v_{i}(x) d x, \quad \forall v \in H^{1}(\Omega) .
\end{gathered}
$$

Here $\stackrel{0}{H}^{1}(\Omega)=\left\{v \in H^{1}(\Omega): v(x)=0, x \in \Gamma_{0}\right\}$ and $H^{1}(\Omega)$ is the Sobolev space of vector functions $v(x)=\left(v_{1}(x), v_{2}(x), v_{3}(x)\right)$, with the norm [1]

$$
\|v\|_{1}:=\left\{\int_{\Omega}\left[|v|^{2}+|\nabla v|^{2}\right] d x\right\}^{1 / 2}, \quad \forall v \in \stackrel{0}{H}^{1}(\Omega) .
$$

We assume that $F \in H^{0}(\Omega)$ and that $f \in H^{0}(\partial \Omega)$.

Denote by $a(u ; u, v) \equiv\langle A u, v\rangle$ the left-hand side and by $l(v)=\langle F, v\rangle+\langle f, v\rangle$ the righthand side of the above integral identity, correspondingly, where $A$ is the plasticity operator, given by (2.5). Then, taking into account the dependence of the plasticity function $g=g\left(e_{\mathrm{i}}^{2}(u)\right)$, on $u \in \stackrel{0}{H}^{1}(\Omega)$, we can rewrite the integral identity (3.1) in the abstract 
functional form (1.5), where the nonlinear functional $a(u ; u, v)$ is defined as follows:

$$
\begin{aligned}
a(u ; v, w)=\int_{\Omega}\{ & {\left[\lambda+\frac{2}{3} \mu g\left(e_{\mathrm{i}}^{2}(u)\right)\right] \theta(v) \theta(w) } \\
& \left.+2 \mu\left[1-g\left(e_{\mathrm{i}}^{2}(u)\right)\right] \varepsilon_{i j}(v) \varepsilon_{i j}(w)\right\} d x, \quad u, v, w \in H^{1}(\Omega) .
\end{aligned}
$$

It is easy to verify that $a(u ; \cdot, \cdot): \stackrel{0}{H}^{1}(\Omega) \times \stackrel{0}{H}^{1}(\Omega) \rightarrow \mathbb{R}^{1}$ is a symmetric bilinear form.

Let us now introduce the functional

$$
J(u)=0.5 \int_{\Omega}\left\{\lambda \theta^{2}(u)+2 \mu \varepsilon_{i j}(u) \varepsilon_{i j}(u)-3 \mu \int_{0}^{e_{\mathrm{i}}^{2}(u)} g(\xi) d \xi\right\} d x, \quad u \in \stackrel{H}{H}^{1}(\Omega) .
$$

Calculating the first Gateaux derivative

$$
\left\langle J^{\prime}(u), v\right\rangle:=\left.\frac{d}{d t} J(u+t v)\right|_{t=0}
$$

of the functional $J(u)$ at $u \in \stackrel{0}{H}^{1}(\Omega)$ on the direction $v \in \stackrel{0}{H}^{1}(\Omega)$, and using the definition $e_{\mathrm{i}}^{2}(u)=(2 / 3) e_{i j}(u) e_{i j}(u)$, we get

$$
\left\langle J^{\prime}(u), v\right\rangle=\int_{\Omega}\left[\lambda \theta(u) \theta(v)+2 \mu \varepsilon_{i j}(u) \varepsilon_{i j}(v)-2 \mu g\left(e_{\mathrm{i}}^{2}(u)\right) e_{i j}(u) e_{i j}(v)\right] d x, \quad \forall u, v \in \stackrel{0}{H}^{1}(\Omega) .
$$

Then we use the identity $e_{i j}(u) e_{i j}(v)=e_{i j}(u) \varepsilon_{i j}(v)$ and rewrite the above integral as follows:

$$
\left\langle J^{\prime}(u), v\right\rangle=\int_{\Omega}\left\{\left[\lambda+\frac{2}{3} \mu g\left(e_{\mathrm{i}}^{2}(u)\right)\right] \theta(u) \theta(v)+2 \mu\left[1-g\left(e_{\mathrm{i}}^{2}(u)\right)\right] \varepsilon_{i j}(u) \varepsilon_{i j}(v)\right\} d x
$$

which shows that $\left\langle J^{\prime}(u), v\right\rangle=a(u ; u, v)$, for all $u, v \in \stackrel{0}{H}^{1}(\Omega)$. Therefore, by definition of the potential, we obtain the following lemma.

Lemma 3.1. The functional $J(u): \stackrel{0}{H}^{1}(\Omega) \rightarrow \mathbb{R}^{1}$, defined by (3.4), is a potential of the plasticity operator $A$, given by (2.5).

Remark 3.2. As we will see below, form (3.3) of the nonlinear functional $a(u ; u, v)$ is also convenient for the iteration process.

It follows from the above lemma that the corresponding functional $\Pi(u)$ defined by (1.7) will be a potential of the problem (2.1)-(2.5).

Now we show that the plasticity operator $A$ is radially continuous (hemicontinuous), that is, the real-valued function

$$
\varphi(t)=\langle A(u+t v), v\rangle, \quad t \in \mathbb{R}
$$

is continuous as a function of $t \in \mathbb{R}$, for any fixed $u, v \in H^{1}(\Omega)$. 
Lemma 3.3. If the plasticity function $g=g(\xi)$ is continuous, then the plasticity operator A, defined by (2.5), is radially continuous.

Proof. Let $\left\{t_{n}\right\} \subset \mathbb{R}$ and $t_{n} \rightarrow t$, as $n \rightarrow \infty$. Then for a fixed $u, v \in \stackrel{0}{H}^{1}(\Omega)$, formula (2.2) implies that

$$
\begin{aligned}
\left\langle A\left(u+t_{n} v\right), v\right\rangle & \\
= & \int_{\Omega}\left[\lambda \theta\left(u+t_{n} v\right) \theta(v)+2 \mu \varepsilon_{i j}\left(u+t_{n} v\right) \varepsilon_{i j}(v)-2 \mu g\left(e_{\mathrm{i}}^{2}\left(u+t_{n} v\right)\right) e_{i j}\left(u+t_{n} v\right) e_{i j}(v)\right] d x \\
= & \langle A(u+t v), v\rangle+\int_{\Omega}\left[\lambda \theta\left(u+\left(t_{n}-t\right) v\right) \theta(v)+2 \mu \varepsilon_{i j}\left(u+\left(t_{n}-t\right) v\right) \varepsilon_{i j}(v)\right] d x \\
& +2 \mu \int_{\Omega}\left\{\left[g\left(e_{\mathrm{i}}^{2}(u+t v)\right)-g\left(e_{\mathrm{i}}^{2}\left(u+t_{n} v\right)\right)\right] e_{i j}(u+t v) e_{i j}(v)\right\} d x \\
& +2 \mu \int_{\Omega}\left\{g\left(e_{\mathrm{i}}^{2}\left(u+t_{n} v\right)\right)\left[e_{i j}(u+t v)-e_{i j}\left(u+t_{n} v\right)\right] e_{i j}(v)\right\} d x .
\end{aligned}
$$

Going to the limit $t_{n} \rightarrow t$ on the right-hand side of the above equality and using continuity of the function $\psi(t)=g\left(e_{\mathrm{i}}^{2}(u+t v)\right)$, we get $\left\langle A\left(u+t_{n} v\right), v\right\rangle \rightarrow\langle A(u+t v), v\rangle$, as $n \rightarrow \infty$.

Lemma 3.4. If the plasticity function $g=g(\xi)$ satisfies the condition (ii), then the plasticity operator $A$ is a strongly monotone one, that is, condition (1.11) holds.

Proof. Based on the equivalence of the strong monotonicity of the potential operator and positivity of its potential, let us calculate the second Gateaux derivative of the functional $J(u)$ defined by (3.4):

$$
\begin{aligned}
J^{\prime \prime}(u ; v, w):=\left.\frac{d}{d t}\left\langle J^{\prime}(u+t w), v\right\rangle\right|_{t=0} \\
=\int_{\Omega}\left\{\left[\lambda+\frac{2}{3} \mu Q\left(e_{\mathrm{i}}^{2}(u)\right)\right] \theta(v) \theta(w)\right. \\
\left.\quad+2 \mu\left[1-Q\left(e_{\mathrm{i}}^{2}(u)\right)\right] \varepsilon_{i j}(v) \varepsilon_{i j}(w)\right\} d x, \quad u, v, w \in H^{1}(\Omega),
\end{aligned}
$$

where $Q\left(e_{\mathrm{i}}^{2}(u)\right)=g\left(e_{\mathrm{i}}^{2}(u)\right)+2 g^{\prime}\left(e_{\mathrm{i}}^{2}(u)\right) e_{\mathrm{i}}^{2}(u)$. Due to condition (ii), $1-Q\left(e_{\mathrm{i}}^{2}(u)\right) \geq \varepsilon_{0}>$ 0 . Then by $\lambda>0$ and Korn's inequality [4], we have

$$
J^{\prime \prime}(u: v, v)=2 \mu \varepsilon_{0} \int_{\Omega} \varepsilon_{i j}(v) \varepsilon_{i j}(v) d x \geq \gamma_{0}\|v\|_{1}^{2}, \quad \forall v \in \stackrel{0}{H}^{1}(\Omega) .
$$

This implies the proof.

Corollary 3.5. Since $A 0=0$, from strong monotonicity of the operator $A$ follows also its coercivity.

Thus the potential operator is radially continuous, strongly monotone, and coercive. Then by the Browder-Minty theorem, we have the following existence theorem. 
Theorem 3.6. Let $g=g(\xi)$ be a piecewise differentiable function satisfying condition (ii). Then the nonlinear boundary value problem (2.1)-(2.5) has a unique solution $u \in \stackrel{0}{H}^{1}(\Omega)$, defined by the integral identity (3.1).

Consider now the nonlinear problem (2.13) related to the bending plate. Let $H^{2}(\Omega)$ be the Sobolev space of functions defined on the domain $\Omega$ with piecewise smooth boundary $\partial \Omega$ and

$$
\stackrel{0}{H}^{2}(\Omega)=\left\{v \in H^{2}(\Omega): u(x)=\frac{\partial u(x)}{\partial n}=0, x \in \partial \Omega\right\} .
$$

Multiplying (2.13) by $v \in \stackrel{0}{H}^{2}(\Omega)$, integrating on $\Omega$, and using boundary conditions, we obtain the following integral identity:

$$
\int_{\Omega} g\left(\xi^{2}(u)\right) H(u, v) d x=\int_{\Omega} F(x) v(x) d x, \quad \forall v \in \stackrel{0}{H^{2}}(\Omega)
$$

where

$$
H(u, v)=\frac{\partial^{2} u}{\partial x_{1}^{2}} \frac{\partial^{2} v}{\partial x_{1}^{2}}+\frac{\partial^{2} u}{\partial x_{2}^{2}} \frac{\partial^{2} v}{\partial x_{2}^{2}}+\frac{\partial^{2} u}{\partial x_{1} \partial x_{2}} \frac{\partial^{2} v}{\partial x_{1} \partial x_{2}}+\frac{1}{2}\left(\frac{\partial^{2} u}{\partial x_{1}^{2}} \frac{\partial^{2} v}{\partial x_{2}^{2}}+\frac{\partial^{2} u}{\partial x_{2}^{2}} \frac{\partial^{2} v}{\partial x_{1}^{2}}\right)
$$

is a bilinear form.

For the function $u \in \stackrel{0}{H}^{2}(\Omega)$ satisfying the integral identity (3.13) for all $v \in \stackrel{0}{H}^{2}(\Omega)$, we define a weak solution of the nonlinear problem (2.13). Taking into account the nonlinear equation (2.13) and formula (2.9), we may define the nonlinear functional $a(u ; u, v)=$ $\langle A u, v\rangle$ as follows:

$$
a(u ; u, v)=\int_{\Omega} g\left(\xi^{2}(u)\right) H(u, v) d x, \quad \forall v \in \stackrel{0}{H}^{2}(\Omega) .
$$

Let us introduce the nonlinear functional

$$
J(u)=\frac{1}{2} \int_{\Omega}\left\{\int_{0}^{\xi^{2}(u)} g(\tau) d \tau\right\} d x, \quad u \in \stackrel{0}{H}^{2}(\Omega)
$$

Lemma 3.7. The fuctional $J(u)$, defined by (3.16), is a potential of the nonlinear operator A, defined by (2.13).

Proof. Calculating the first Gateaux derivative

$$
\left\langle J^{\prime}(u), v\right\rangle:=\left.\frac{d}{d t} J(u+t v)\right|_{t=0}, \quad u, v \in \stackrel{0}{H^{2}}(\Omega),
$$


of the functional $J(u)$ at $u \in \stackrel{0}{H}^{2}(\Omega)$ and on $v \in \stackrel{0}{H}^{2}(\Omega)$, we obtain

$$
\begin{aligned}
\left\langle J^{\prime}(u), v\right\rangle & =\frac{1}{2} \int_{\Omega}\left\{\frac{d}{d t}\left[\int_{0}^{\xi^{2}(u+t v)} g(\tau) d \tau\right]_{t=0}\right\} d x \\
& =\frac{1}{2} \int_{\Omega}\left\{g\left(\xi^{2}(u+t v)\right) \cdot \frac{d}{d t} \xi^{2}(u+t v)\right\}_{t=0} d x .
\end{aligned}
$$

On the other hand, due to (2.9),

$$
\begin{aligned}
& \frac{d}{d t}\left[\xi^{2}(u+t v)\right]_{t=0} \\
& \quad=2\left[\frac{\partial^{2} u}{\partial x_{1}^{2}} \frac{\partial^{2} v}{\partial x_{1}^{2}}+\frac{\partial^{2} u}{\partial x_{2}^{2}} \frac{\partial^{2} v}{\partial x_{2}^{2}}+\frac{\partial^{2} u}{\partial x_{1} \partial x_{2}} \frac{\partial^{2} v}{\partial x_{1} \partial x_{2}}+\frac{1}{2}\left(\frac{\partial^{2} u}{\partial x_{1}^{2}} \frac{\partial^{2} v}{\partial x_{2}^{2}}+\frac{\partial^{2} u}{\partial x_{2}^{2}} \frac{\partial^{2} v}{\partial x_{1}^{2}}\right)\right] .
\end{aligned}
$$

Hence, taking into account (3.14), we get

$$
\frac{d}{d t}\left[\xi^{2}(u+t v)\right]_{t=0}=2 H(u, v) .
$$

Thus,

$$
\left\langle J^{\prime}(u), v\right\rangle=\int_{\Omega} g\left(\xi^{2}(u)\right) H(u, v) d x,
$$

and by definition (3.15), we obtain the proof.

Let us now analyze monotonicity of the nonlinear bending operator $A$. For this aim, we introduce the energy norm

$$
\|v\|_{E}:=\left\{\int_{\Omega} H(v, v) d x\right\}^{1 / 2}
$$

and compare this norm with the seminorm

$$
|v|_{2}:=\left\{\int_{\Omega}\left[\left(\frac{\partial^{2} v}{\partial x_{1}^{2}}\right)^{2}+\left(\frac{\partial^{2} v}{\partial x_{2}^{2}}\right)^{2}+\left(\frac{\partial^{2} v}{\partial x_{1} \partial x_{2}}\right)^{2}\right] d x\right\}^{1 / 2}
$$

of the space $H^{2}(\Omega)$.

Lemma 3.8. If $v \in \stackrel{0}{H}^{2}(\Omega)$, then

$$
|v|_{2}^{2} \leq\|v\|_{E}^{2} \leq 2|v|_{2}^{2}
$$

that is, the energy norm $|\cdot|_{2}$ and the seminorm $\|\cdot\|_{E}$ are equivalent.

Proof. First note that for all $v \in \stackrel{0}{H}^{2}(\Omega)$, the following formula is valid [14]:

$$
\int_{\Omega}\left[\left(\frac{\partial^{2} v}{\partial x_{1} \partial x_{2}}\right)^{2}-\frac{\partial^{2} v}{\partial x_{1}^{2}} \frac{\partial^{2} v}{\partial x_{2}^{2}}\right] d x=0
$$


12 Monotonicity of nonlinear problems

Then taking into account (3.14), we have

$$
\begin{aligned}
\int_{\Omega} H(v, v) d x= & \int_{\Omega}\left[\left(\frac{\partial^{2} v}{\partial x_{1}^{2}}\right)^{2}+\left(\frac{\partial^{2} v}{\partial x_{2}^{2}}\right)^{2}+\left(\frac{\partial^{2} v}{\partial x_{1} \partial x_{2}}\right)^{2}+\frac{\partial^{2} v}{\partial x_{1}^{2}} \frac{\partial^{2} v}{\partial x_{2}^{2}}\right] d x \\
& +\int_{\Omega}\left[\left(\frac{\partial^{2} v}{\partial x_{1}^{2}}\right)^{2}+\left(\frac{\partial^{2} v}{\partial x_{2}^{2}}\right)^{2}+2\left(\frac{\partial^{2} v}{\partial x_{1} \partial x_{2}}\right)^{2}\right] d x \\
\geq & \int_{\Omega}\left[\left(\frac{\partial^{2} v}{\partial x_{1}^{2}}\right)^{2}+\left(\frac{\partial^{2} v}{\partial x_{2}^{2}}\right)^{2}+\left(\frac{\partial^{2} v}{\partial x_{1} \partial x_{2}}\right)^{2}\right] d x \\
= & |v|_{2}^{2} .
\end{aligned}
$$

On the other hand,

$$
\int_{\Omega} H(v, v) d x \leq 2 \int_{\Omega}\left[\left(\frac{\partial^{2} v}{\partial x_{1}^{2}}\right)^{2}+\left(\frac{\partial^{2} v}{\partial x_{2}^{2}}\right)^{2}+\left(\frac{\partial^{2} v}{\partial x_{1} \partial x_{2}}\right)^{2}\right] d x=|v|_{2}^{2} .
$$

These two estimates imply (3.24).

By using the equivalence of the norm $\|\cdot\|_{2}$ and the seminorm $|\cdot|_{2}$ in $\stackrel{0}{H}^{2}(\Omega)$ [17], we obtain the following corollary.

Corollary 3.9. If $v \in \stackrel{0}{H}^{2}(\Omega)$, then

$$
\exists \alpha_{1}, \alpha_{2}>0, \quad \alpha_{1}\|v\|_{2} \leq\|v\|_{E} \leq \alpha_{2}\|v\|_{2},
$$

that is, the $H^{2}$-norm and the energy norm are equivalent.

The lemma permits one to obtain also the following upper estimate.

Corollary 3.10. If $u, v \in \stackrel{0}{H}^{2}(\Omega)$, then

$$
\int_{\Omega}|H(u, v)| d x \leq \alpha_{2}^{2}\|u\|_{2} \cdot\|v\|_{2}
$$

Proof. We use the Schwartz inequality in the following form:

$$
(H(u, v))^{2} \leq H(u, u) H(v, v) .
$$

Then taking into account (3.28), we obtain

$$
\begin{aligned}
\int_{\Omega}|H(u, v)| d x & \leq \int_{\Omega}(H(u, u))^{1 / 2}(H(v, v))^{1 / 2} \\
& \leq\left(\int_{\Omega}|H(u, u)| d x\right)^{1 / 2}\left(\int_{\Omega}|H(v, v)| d x\right)^{1 / 2} \\
& \leq \alpha_{2}^{2}\|u\|_{2}\|v\|_{2} .
\end{aligned}
$$


By using these auxiliary results, we can prove the strong monotonicity in $\stackrel{0}{H}^{2}(\Omega)$ of the potential operator $A$, defined by (2.13).

Lemma 3.11. If the plasticity function $g=g\left(\xi^{2}\right)$ satisfies conditions (i1)-(i4), then the potential operator $A$ is strongly monotone in $\stackrel{0}{H}^{2}(\Omega)$, that is,

$$
\forall u, v \in \stackrel{0}{H}^{2}(\Omega), \quad\langle A u-A v, u-v\rangle \geq \gamma_{1}\|u-v\|_{2}^{2}, \quad \gamma_{1}>0 .
$$

Proof. Consider the second Gateaux derivative of the functional $J(u)$, defined by $(3.16)$ :

$$
\begin{aligned}
\left\langle J^{\prime \prime}(u), v, w\right\rangle: & =\left.\frac{d}{d t}\left\langle J^{\prime}(u+t w), v\right\rangle\right|_{t=0} \\
& =\frac{d}{d t}\left\{\int_{\Omega} g\left(\xi^{2}(u+t w)\right) H(u+t w, v) d x\right\}_{t=0} \\
& =\left\{\int_{\Omega}\left[2 g^{\prime}\left(\xi^{2}(u+t w)\right) H(u, w) H(u+t w, v)+g\left(\xi^{2}(u+t w)\right) H(w, v)\right] d x\right\}_{t=0} \\
& =\int_{\Omega}\left[2 g^{\prime}\left(\xi^{2}(u)\right) H(u, w) H(u, v)+g\left(\xi^{2}(u)\right) H(w, v)\right] d x .
\end{aligned}
$$

For $w=v$, we have $\left(H(v, v)=\xi^{2}(v)\right)$,

$$
\left\langle J^{\prime \prime}(u), v, v\right\rangle:=\int_{\Omega}\left[g\left(\xi^{2}(u)\right) \xi^{2}(v)+2 g^{\prime}\left(\xi^{2}(u)\right) H^{2}(u, v)\right] d x
$$

Condition (i2) with the Schwartz inequality $(H(u, v))^{2} \leq H(u, u) H(v, v)$ implies that

$$
\begin{aligned}
\left\langle J^{\prime \prime}(u), v, v\right\rangle & \geq \int_{\Omega}\left[g\left(\xi^{2}(u)\right) \xi^{2}(v)+2 g^{\prime}\left(\xi^{2}(u)\right) \xi^{2}(u) \xi^{2}(v)\right] d x \\
& \left.=\int_{\Omega}\left[g\left(\xi^{2}(u)\right)+2 g^{\prime}\left(\xi^{2}(u)\right) \xi^{2}(u)\right] \xi^{2}(v)\right] d x .
\end{aligned}
$$

By using the condition (i3) on the right-hand side and applying Corollary 3.10, finally we get

$$
\left\langle J^{\prime \prime}(u), v, v\right\rangle \geq c_{2} \int_{\Omega} \xi^{2}(v) d x=\int_{\Omega} H(v, v) d x \geq \alpha_{1} c_{2}\|v\|_{2}^{2}
$$

The positivity of the second Gateaux derivative of the functional $J(u)$ means that the operator $A$ is a strongly monotone one.

Corollary 3.12. Since $A \theta=\theta$, where $\theta \in \stackrel{0}{H}^{2}(\Omega)$ is a zero element, the monotonicity of the nonlinear operator $A$ also means its coercivity

$$
\langle A v, v\rangle \geq \gamma_{1}\|v\|_{2}^{2}, \quad \gamma_{1}>0 .
$$

Lemma 3.13. The nonlinear operator A, given by (2.13), is radially continuous (hemicontinuous). 
The proof of this lemma is the same as proof of Lemma 3.3.

Thus, the potential operator $A$ for a bending plate is a radially continuous, strongly monotone, and coercive one. By the Browder-Minty theorem, we get the following theorem.

Theorem 3.14. If conditions (i1)-(i3) hold, then the nonlinear problem (2.13) has a unique solution in $\stackrel{0}{H}^{2}(\Omega)$, defined by the integral identity (3.13).

Finally consider the nonlinear problem of elastoplastic torsion of a cylindrical bar. The weak solution $u \in \stackrel{0}{H}^{1}(\Omega):=\left\{u(x) \in H^{1}(\Omega): u(x)=0, x \in \partial \Omega\right\}$ of problem (2.14) is defined as follows:

$$
\int_{\Omega} g\left(|\nabla u|^{2}\right) \nabla u \nabla v d x=2 \int_{\Omega} \Theta(x) v(x) d x, \quad \forall v \in \stackrel{0}{H}^{1}(\Omega),
$$

where

$$
\xi^{2}(u)=|\nabla u|^{2}, \quad \nabla u \nabla v=\frac{\partial u}{\partial x_{1}} \frac{\partial v}{\partial x_{1}}+\frac{\partial u}{\partial x_{2}} \frac{\partial v}{\partial x_{2}} .
$$

Introducing the nonlinear functional

$$
J(u)=\frac{1}{2} \int_{\Omega}\left\{\int_{0}^{\xi^{2}(u)} g(\tau) d \tau\right\} d x, \quad u \in \stackrel{0}{H}^{1}(\Omega),
$$

and calculating its Gateaux derivative, we obtain

$$
\left\langle J^{\prime}(u), v\right\rangle=\int_{\Omega} g\left(|\nabla u|^{2}\right) \nabla u \nabla v d x, \quad \forall v \in \stackrel{0}{H^{1}}(\Omega) .
$$

Hence, the nonlinear functional (3.40) is a potential for the operator $A$, given by (2.14). We can also easily prove that this operator is hemicontinuous. Thus, to apply the BrowderMinty theorem, we only need to prove strong monotonicity of the operator $A$. For this aim, let us estimate the second Gateaux derivative of the functional $J(u)$, defined by (3.40). We have

$$
\left\langle J^{\prime \prime}(u), v, v\right\rangle=\int_{\Omega}\left[g\left(|\nabla u|^{2}\right)+2 g^{\prime}\left(|\nabla u|^{2}\right)|\nabla u|^{2}\right]|\nabla v|^{2} d x \geq c_{2}\|\nabla v\|_{0}^{2}, \quad \forall v \in H^{1}(\Omega),
$$

due to the condition (i2). Here $\|\cdot\|_{0}$ is the norm of the space $H^{0}(\Omega) \equiv L_{2}(\Omega)$-norm. For the function $v \in \stackrel{0}{H}^{1}(\Omega)$ by using the Poincare inequality [16]

$$
\|v\|_{0}^{2} \leq c_{\Omega}\|\nabla v\|_{0}^{2}
$$

we get

$$
\|\nabla v\|_{0}^{2}=\sigma\|\nabla v\|_{0}^{2}+(1-\sigma)\|\nabla v\|_{0}^{2} \geq \sigma c_{\Omega}^{-2}\|v\|_{0}^{2}+(1-\sigma)\|v\|_{0}^{2}
$$


Equating the coefficients $\left(\sigma c_{\Omega}^{-2}=1-\sigma\right)$, we define the free parameter as follows: $\sigma=$ $c_{\Omega}^{2} /\left(1+c_{\Omega}^{2}\right)$. Hence

$$
\left\langle J^{\prime \prime}(u), v, v\right\rangle \geq \gamma_{1}\|v\|_{1}^{2}, \quad \gamma_{1}=\frac{c_{\Omega}^{2}}{1+c_{\Omega}^{2}},
$$

which gives the strong monotonicity of the nonlinear operator $A$, given by (2.14). Applying the Browder-Minty theorem to the nonlinear problem of elastoplastic torsion of a cylindrical bar, we get the following theorem.

THeOREM 3.15. If conditions (i1)-(i4) hold, then the nonlinear problem (2.14) has a unique solution in $\stackrel{0}{H}^{2}(\Omega)$, defined by the integral identity (3.38).

\section{Sufficient condition for the convexity argument: convergence results}

Based on the abstract iteration scheme (1.8), we realize the linearization of the nonlinear problems (3.1), (3.13), and (3.38) correspondingly, as follows:

$$
\begin{gathered}
\int_{\Omega}\left\{\left[\lambda+\frac{2}{3} \mu g\left(e_{\mathrm{i}}^{2}\left(u^{(n-1)}\right)\right)\right] \theta\left(u^{(u)}\right) \theta(v)+2 \mu\left[1-g\left(e_{\mathrm{i}}^{2}\left(u^{(n-1)}\right)\right)\right] \varepsilon_{i j}\left(u^{(n)}\right) \varepsilon_{i j}(v)\right\} d x \\
=\int_{\Omega} F_{i}(x) v_{i}(x) d x+\int_{\Gamma_{1}} f_{i}(x) v_{i}(x) d x, \quad \forall v=\left(v_{1}, v_{2}, v_{3}\right) \in H^{1}(\Omega), \\
\int_{\Omega} g\left(\xi^{2}\left(u^{(n-1)}\right)\right) H\left(u^{(n)}, v\right) d x=\int_{\Omega} F(x) v(x) d x, \quad \forall v \in H^{2}(\Omega), \\
\int_{\Omega} g\left(\left|\nabla u^{(n-1)}\right|^{2}\right) \nabla u^{(n)} \nabla v d x=2 \int_{\Omega} \Theta(x) v(x) d x, \quad \forall v \in H^{1}(\Omega),
\end{gathered}
$$

where $n=1,2,3, \ldots$, and $u^{(0)}$ is an initial iteration chosen for each of the problems, respectively.

As it was noted in the introduction, a necessary condition for convergence of each iteration process is fulfillment of the convexity argument (1.9). In this case, the rate of convergence for each of the above problems is estimated via the potentials as in (1.10). Here, we are going to obtain a necessary condition for fulfillment of the convexity argument (1.9).

Consider first the plasticity operator (2.5).

LEMma 4.1. If the differentiable function $g=g(\xi)$ satisfies the condition (iii), then the convexity argument (1.9) holds for the plasticity operator A, defined by (2.5).

Proof. Taking into account the last integral in (3.4), we introduce the function

$$
Q(t)=\int_{0}^{t} g(\tau) d \tau
$$

Due to the condition (iii), $Q^{\prime \prime}(t)=g^{\prime}(t)>0$, and $Q=Q(t)$ is a convex function. Hence by the well-known property of convex functions,

$$
Q^{\prime}\left(t_{1}\right)\left(t_{2}-t_{1}\right)-Q\left(t_{2}\right)+Q\left(t_{1}\right) \leq 0, \quad \forall t_{2}>t_{1}>0 .
$$


Substituting $t_{1}=e_{\mathrm{i}}^{2}(u), t_{2}=e_{\mathrm{i}}^{2}(v)$, we get

$$
g\left(e_{\mathrm{i}}^{2}(u)\right)\left[g\left(e_{\mathrm{i}}^{2}(u)\right)-g\left(e_{\mathrm{i}}^{2}(v)\right)\right]+\int_{e_{\mathrm{i}}^{2}(u)}^{e_{\mathrm{i}}^{2}(v)} g(\tau) d \tau \geq 0, \quad \forall u, v \in \stackrel{H}{H}^{1}(\Omega) .
$$

Let us now calculate the left-hand side of (1.9), by using (3.3) and (3.4):

$$
\begin{aligned}
& 0.5 a(u ; v, v)-0.5 a(u ; u, u)-J(v)+J(u) \\
& \quad=\mu \int_{\Omega} g\left(e_{\mathrm{i}}^{2}(u)\right)\left[e_{i j}(u) e_{i j}(u)-e_{i j}(v) e_{i j}(v)\right] d x+\frac{2}{3} \mu \int_{\Omega}\left\{\int_{e_{\mathrm{i}}^{2}(u)}^{e_{\mathrm{i}}^{2}(v)} g(\tau) d \tau\right\} d x .
\end{aligned}
$$

Taking into account the identity $e_{\mathrm{i}}^{2}(u)=(2 / 3) e_{i j}(u) e_{i j}(u)$, we get

$$
\begin{aligned}
& 0.5 a(u ; v, v)-0.5 a(u ; u, u)-J(v)+J(u) \\
& \quad=\frac{2}{3} \mu \int_{\Omega}\left\{g\left(e_{\mathrm{i}}^{2}(u)\right)\left[e_{i j}(u) e_{i j}(u)-e_{i j}(v) e_{i j}(v)\right]+\int_{e_{\mathrm{i}}^{2}(u)}^{e_{\mathrm{i}}^{2}(v)} g(\tau) d \tau\right\} d x .
\end{aligned}
$$

Using here inequality (4.6), we obtain the proof.

Consider now the nonlinear operator $A$ for a bending plate.

Lemma 4.2. If the differentiable function $g=g(\xi)$ satisfies the condition (i3), then the convexity argument (1.9) holds for the plasticity operator A, defined by (2.13).

Proof. Based on definitions (3.15) and (3.16), we calculate the left-hand side of inequality (1.9):

$$
\begin{aligned}
0.5 a(u ; v, v)-0.5 a(u ; u, u)-J(v)+J(u) & \\
= & 0.5 \int_{\Omega} g\left(\xi^{2}(u)\right) H(v, v) d x-0.5 \int_{\Omega} g\left(\xi^{2}(u)\right) H(u, u) d x \\
& -0.5 \int_{\Omega}\left\{\int_{0}^{\xi^{2}(v)} g(\tau) d \tau\right\} d x+0.5 \int_{\Omega}\left\{\int_{0}^{\xi^{2}(u)} g(\tau) d \tau\right\} d x \\
= & 0.5 \int_{\Omega}\left\{g\left(\xi^{2}(u)\right)\left[\xi^{2}(v)-\xi^{2}(u)\right]+\int_{\xi^{2}(v)}^{\xi^{2}(u)} g(\tau) d \tau\right\} d x,
\end{aligned}
$$

for all $u, v \in \stackrel{0}{H}^{2}(\Omega)$. We introduce again function (4.4) and observe that this function is a concave one, due to the condition $g^{\prime}(\xi)<0$. Hence,

$$
Q^{\prime}\left(t_{1}\right)\left(t_{2}-t_{1}\right)-Q\left(t_{2}\right)+Q\left(t_{1}\right) \geq 0, \quad \forall t_{2}>t_{1}>0
$$

Substituting here $t_{1}=\xi^{2}(u)$ and $t_{2}=\xi^{2}(v)$, we get

$$
g\left(\xi^{2}(u)\right)\left[g\left(\xi^{2}(v)\right)-g\left(\xi^{2}(u)\right)\right]+\int_{\xi^{2}(u)}^{\xi^{2}(v)} g(\tau) d \tau \geq 0 .
$$

Substituting this on the right-hand side of (4.9), we obtain the proof. 
The same result is valid for the problem of elastoplastic torsion, given by (3.38).

Note that all conditions (i)-(iii), as well as (i1)-(i4), were obtained from the main assumptions (a1)-(a3) of the deformation theory. Hence, summarizing the above results related to iteration schemes $(4.1)-(4.3)$, we can formulate the following convergence theorem.

Theorem 4.3. If the assumptions (a1)-(a3) of the deformation theory hold, then each $\left\{u^{(n)}(x)\right\}$ sequence of approximate solutions obtained by iteration schemes (4.1)-(4.3) converges to the corresponding solutions of nonlinear problems (3.1), (3.13), and (3.38), respectively. Moreover, the rate of convergence is estimated by (1.10), via potentials of each problem.

According to [7, Lemma 1], if the convexity argument (1.9) holds, then the numerical sequence $\left\{\Pi\left(u^{(n)}\right)\right\}$, where iterations $\left\{u^{(n)}(x)\right\}$ are defined from the abstract iteration scheme (1.8), is a monotone decreasing one. Hence, the sequences $\left\{\Pi\left(u^{(n)}\right)\right\}$, defined in all the above iteration schemes (4.1)-(4.3), are monotone decreasing, which precisely agrees with the physical sense of the plasticity problems.

\section{Acknowledgment}

This work was supported by Scientific Research Foundation of Kocaeli University.

\section{References}

[1] R. A. Adams, Sobolev Spaces, Pure and Applied Mathematics, vol. 65, Academic Press, New York, 1975.

[2] J. H. Argyris and S. Kelsey, Energy Theorems and Structural Analysis, Butterworths, London, 1960.

[3] P. G. Ciarlet, The Finite Element Method for Elliptic Problems, Studies in Mathematics and Its Applications, vol. 4, North-Holland, Amsterdam, 1978.

[4] G. Duvaut and J.-L. Lions, Inequalities in Mechanics and Physics, Grundlehren der mathematischen Wissenschaften, vol. 219, Springer, Berlin, 1976.

[5] H. Gajewski, K. Gröger, and K. Zacharias, Nichtlineare Operatorgleichungen und Operatordifferentialgleichungen, Mathematische Lehrbücher und Monographien, II. Abteilung, Mathematische Monographien, vol. 38, Akademie, Berlin, 1974.

[6] A. Hasanov, An inverse problem for an elastoplastic medium, SIAM Journal on Applied Mathematics 55 (1995), no. 6, 1736-1752.

[7] Convexity argument for monotone potential operators and its application, Nonlinear Analysis. Theory, Methods \& Applications. An International Multidisciplinary Journal. Series A: Theory and Methods 41 (2000), no. 7-8, 907-919.

[8] A. Hasanov and Z. Seyidmamedov, Determination of unknown elastoplastic properties in Signorini problem, International Journal of Non-Linear Mechanics 33 (1998), no. 6, 979-991.

[9] A. A. Il'yušin, Plasticity. Part 1: Elasticity-Plastic Deformations, OGIZ, Moscow, 1948.

[10] L. M. Kachanov, Fundamentals of the Theory of Plasticity, Mir, Moscow, 1974.

[11] O. A. Ladyzhenskaya, The Boundary Value Problems of Mathematical Physics, Applied Mathematical Sciences, vol. 49, Springer, New York, 1985.

[12] A. Langenbach, Elastisch-plastische deformationen von platten, Zeitschrift für Angewandte Mathematik und Mechanik. Ingenieurwissenschaftliche Forschungsarbeiten 41 (1961), 126-134 (German). 


\section{Monotonicity of nonlinear problems}

[13] _ Verallgemeinerte und exakte Lösungen des Problems der elastisch-plastischen Torsion von Stäben, Mathematische Nachrichten 28 (1964/1965), 219-234 (German).

[14] S. G. Mikhlin, Variational Methods in Mathematical Physics, Macmillan, New York, 1964.

[15] J. Nečas and I. Hlaváček, Mathematical Theory of Elastic and Elasto-Plastic Bodies: An Introduction, Elsevier, Amsterdam, 1980.

[16] R. E. Showalter, Monotone Operators in Banach Space and Nonlinear Partial Differential Equations, Mathematical Surveys and Monographs, vol. 49, American Mathematical Society, Rhode Island, 1997.

[17] K. Washizu, Variational Methods in Elasticity and Plasticity, 2nd ed., Pergamon, New York, 1981.

Alemdar Hasanov: Applied Mathematical Sciences Research Center and

Department of Mathematics, Kocaeli University, Ataturk Bulvari, 41300 Izmit,

Kocaeli, Turkey

E-mail address: ahasanov@kou.edu.tr 


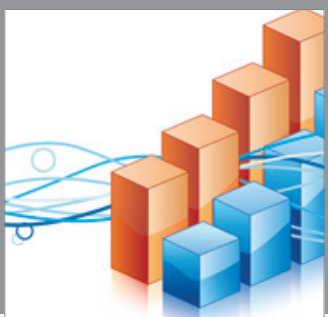

Advances in

Operations Research

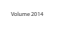

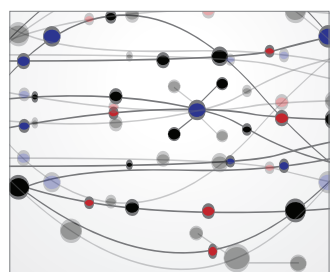

\section{The Scientific} World Journal
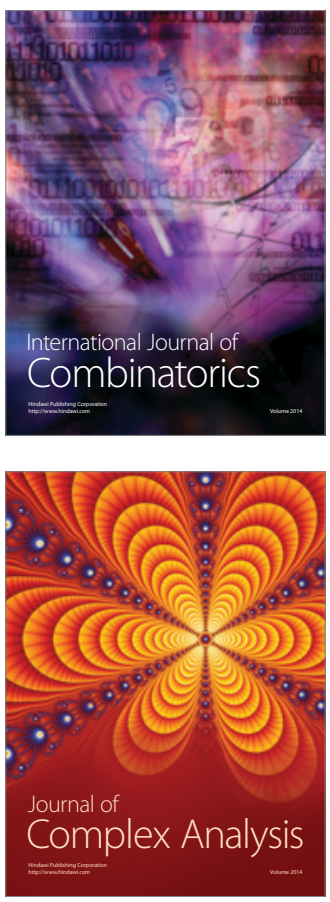

International Journal of

Mathematics and

Mathematical

Sciences
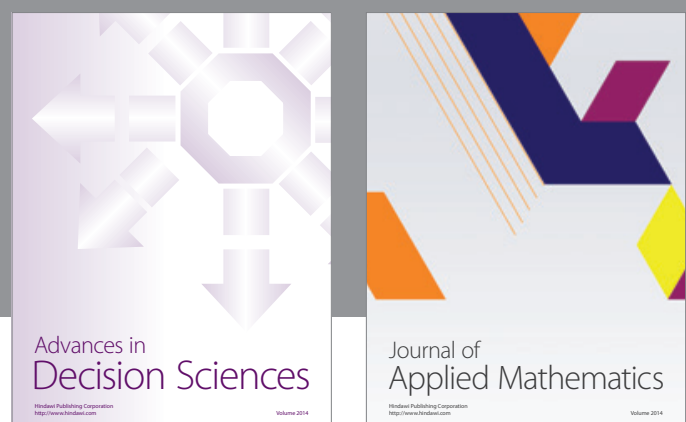

Journal of

Applied Mathematics
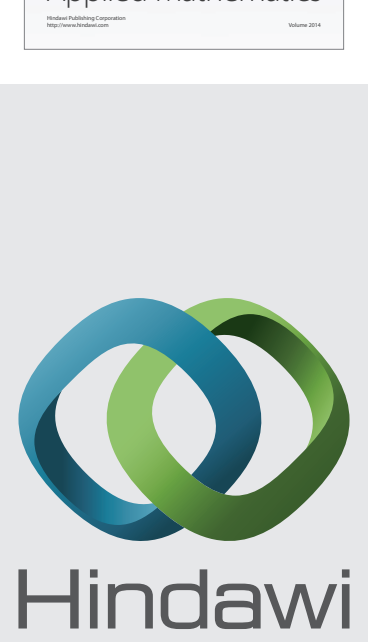

Submit your manuscripts at http://www.hindawi.com
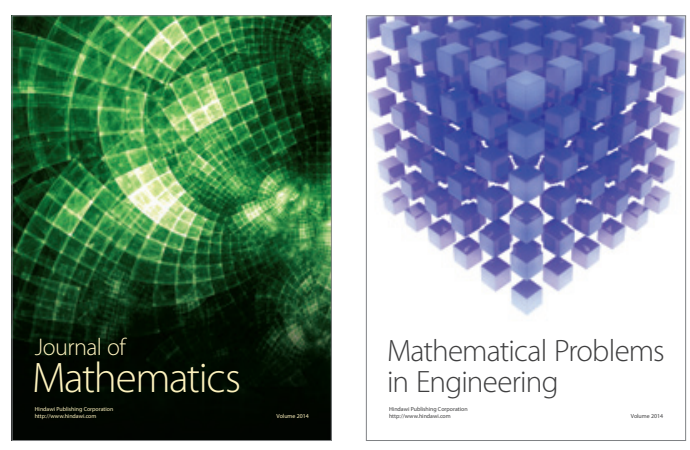

Mathematical Problems in Engineering
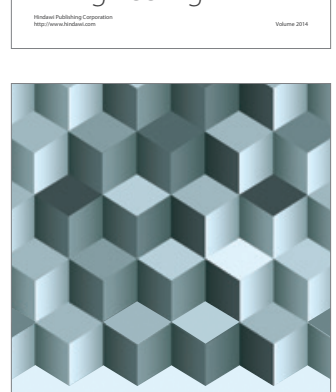

Journal of

Function Spaces
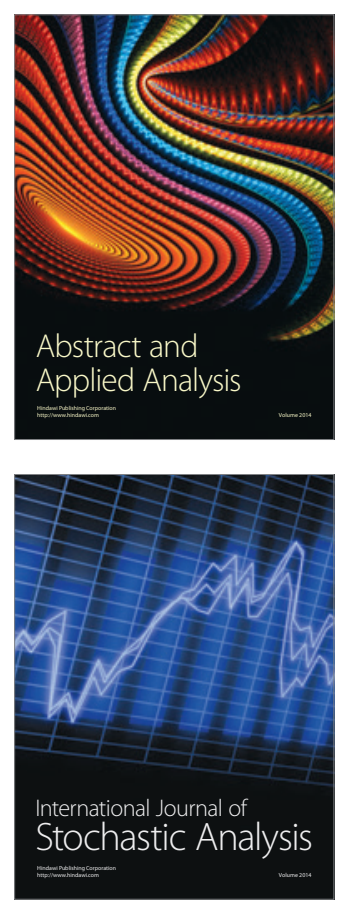

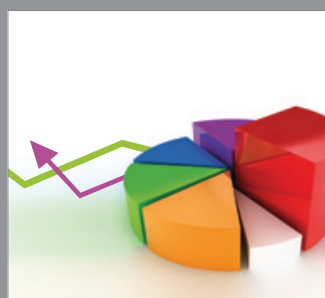

ournal of

Probability and Statistics

Promensencen
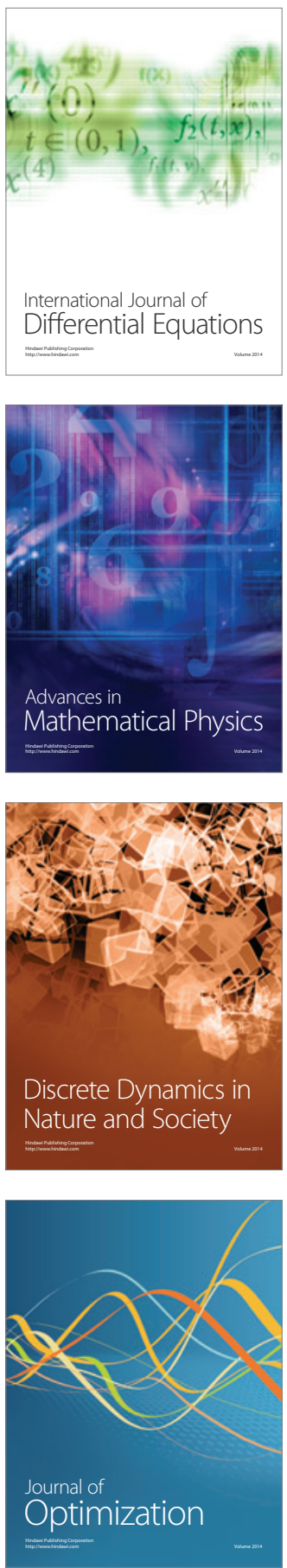